\title{
Modeling, Control Design, and Experimental Validation of an Overactuated Thermal Management System for Engine Dynamometer Applications
}

\author{
Chris Vermillion, Jing Sun, Fellow, IEEE, and Ken Butts
}

\begin{abstract}
Effective engine mapping and calibration are contingent upon tight control of the environment in which the mapping and calibration are performed. Among the most important variables to be controlled are the temperatures of coolant and oil that circulate through the engine block. Because of the large time constants associated with thermodynamic systems, controlling these variables often represents a bottleneck in the engine mapping and calibration processes. In this paper, we examine a particular layout for a thermal management unit, which is currently being used in practice. By developing and analyzing a thermodynamic model of the system, we are able to gain insight into the system dynamics and explore special features to optimize the temperature response. In particular, we will show how the overactuation in the system may be leveraged in the presence of hard saturation constraints and different dynamic actuator authorities. We present design and validation results (both simulation and experimental) for the proposed controller, and compare the performance to the baseline controller in order to quantify improvements.
\end{abstract}

Index Terms-Automotive control, calibration, optimal control, overactuated systems, powetrain control.

\section{INTRODUCTION}

$\mathbf{E}$ NGINE mapping refers to the process where models characterizing the relations between the engine inputs (e.g., spark timing, air charge, fuel, valve timing, etc., for gasoline engines) and outputs (e.g., torque, exhaust temperature, emissions, etc.) are developed and validated. A controlled environment such as an engine test cell with a dynamometer has been used for mapping to ensure that these models are accurate. One key control system that facilitates this process is the thermal management system, which controls the temperature of the coolant and oil that pass through the engine block. For effective engine mapping and calibration, it is generally agreed that these fluid temperatures at the engine outlet should be maintained to within $2^{\circ} \mathrm{C}$ of the desired setpoint, and tighter temperature control is always desirable. For this application, the main focus is on temperature regulation, while other poten-

Manuscript received March 06, 2007. Manuscript received in final form July 06, 2007. First published December 31, 2008; current version published April 24, 2009. Recommended by Associate Editor P. Meckl.

C. Vermillion is with the University of Michigan, EECS/NAME, Ann Arbor, MI 48109 USA (e-mail: cvermill@umich.edu).

J. Sun is with the Electrical Engineering-Systems and Naval Architecture/Marine Engineering, University of Michigan, Ann Arbor, MI 48109 USA (e-mail: jingsun@umich.edu).

$\mathrm{K}$. Butts is with Toyota Engineering and Manufacturing-North America, BR-MBC, Ann Arbor, MI 48109 USA (e-mail: ken.butts@tema.toyota.com).

Color versions of one or more of the figures in this paper are available online at http://ieeexplore.ieee.org.

Digital Object Identifier 10.1109/TCST.2008.2001267

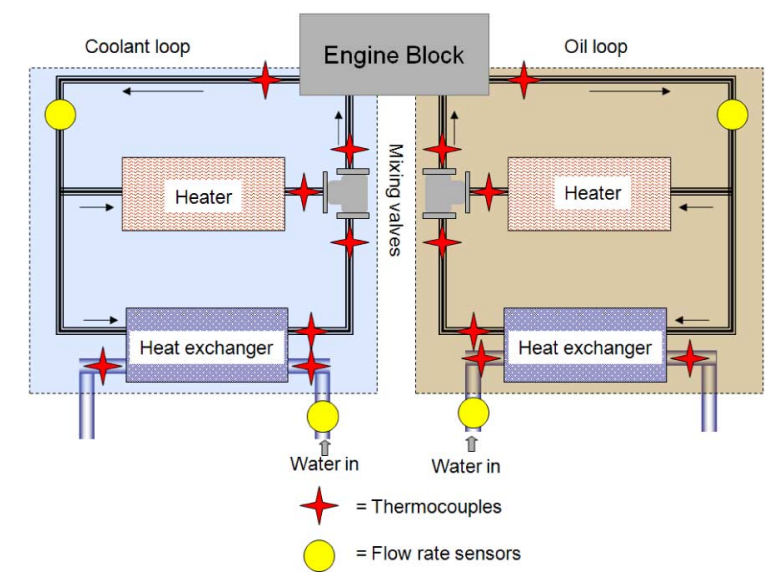

Fig. 1. System diagram.

tial performance indices, such as actuator power consumption (which is very small compared to the power used in running the entire test cell), are neglected. Therefore, the performance of a thermal management system studied in this paper will be measured by two performance metrics: the percent of testing time spent within 2 degrees of setpoint and the root mean squared error (RMSE) between setpoint and actual temperature.

A hardware configuration of the thermal management system that supports engine testing is illustrated in Fig. 1. The system consists of a coolant loop and an oil loop, each of which has a heat exchanger, a heater, and a mixing valve. The heat exchanger cools the fluid by exchanging heat between the working fluid (either coolant or oil) and cooling water that is supplied by the building water system. In order to provide supplemental heating to the fluid, which is necessary when the engine is running at low speed and load, a heater is incorporated. The heat exchanger and heater are arranged in parallel, and these two flows are mixed together in a proportion specified by the mixing valve, as illustrated in Fig. 1. For each loop (coolant and oil), the hardware, which includes the mixing valve, heat exchanger, and heater, is housed inside a box of dimension approximately $2 \mathrm{~m}$ $\times 1 \mathrm{~m} \times 1 \mathrm{~m}$. The mixing valve and heater are the two actuators, whereas the coolant and oil flow rates are dictated by the engine coolant and oil pumps and vary proportionally with engine speed, and the flow rate of cooling water is governed by an external building water pump, which is not under the control of the test cell.

Several other systems similar to Fig. 1 have been studied in the literature, with models developed and control strategies proposed. For example, [1]-[4] consider systems without a supple- 
mental heater in parallel with the heat exchanger. In [5], Setlur $e t$ al. propose a nonlinear control strategy for an actual in-vehicle system, which includes an auxiliary heater in the main loop, immediately before the engine. Temperature dynamics of the engine block and heater coil are treated as sources of unmeasurable disturbances. In [6], Zou et al. present a model similar to the one presented in this paper, but place emphasis on simulating the cooling system performance in the presence of a thermostat that exhibits hysteresis. In [7] and [8], the authors assess the theoretical performance of cooling systems with electronically actuated mixing valves. This paper adds to existing models by taking into consideration the temperature dynamics of the solid heater coil and engine block, rather than just considering the fluids and assuming immediate heat transfer through these solid elements. This consideration yields important dynamic properties, detailed in [9], that will be leveraged in this work to optimally control the system, especially in the presence of actuator saturation limits and different dynamic authorities. As a benchmark reference, the baseline control strategy that was previously used will be included in this paper. This control strategy, which is detailed in Section V, consists of an "all-purpose," off-the-shelf proportional-integral-differential (PID) controller, which typically uses only one of the two actuators at a given time. The mixing valve is used to cool the fluid while the heater is used when the temperature is below the setpoint and heating is required. This controller structure greatly limits the performance of the system, especially when the heater is the only active control input, due to the large thermal inertia and consequent poor dynamic control authority. The result is a temperature response that is often slow and exhibits poor tracking around the desired temperature. This leads to an inefficient engine mapping and calibration process, wherein a large amount of time is spent waiting for temperatures to settle rather than collecting useful data. The controller proposed in this paper overcomes the drawbacks of the baseline controller with a model-based feedforward/feedback combination. The novelty of the proposed design is in the feedforward control, where the feedforward control inputs, referred to as actuator settings in this work, are determined by evaluating and optimizing the transient tracking and disturbance rejection capabilities of the system over all feasible candidate settings, under saturation constraints. Simulation and experimental results show that this design yields a significant performance advantage over the baseline design.

This paper is organized as follows. In Section II, a dynamic model for the system is developed, the system identification process is discussed, and model validation results are presented. Dynamic analysis results are given in Section III, which lead to the control design process of Section IV, where the overactuation of the system is exploited for optimal performance. A novel feedforward control strategy is used in order to maximize transient control authority, thereby facilitating an effective feedback control design. Additionally, an implementation strategy, using support vector classification, is proposed in order to practically execute the proposed feedforward algorithm. To illustrate the effectiveness of the proposed controller, simulation and experimental validation results are included and compared to the baseline control performance in Section V. Finally, in Section VI, system uncertainties due to varying engine char-

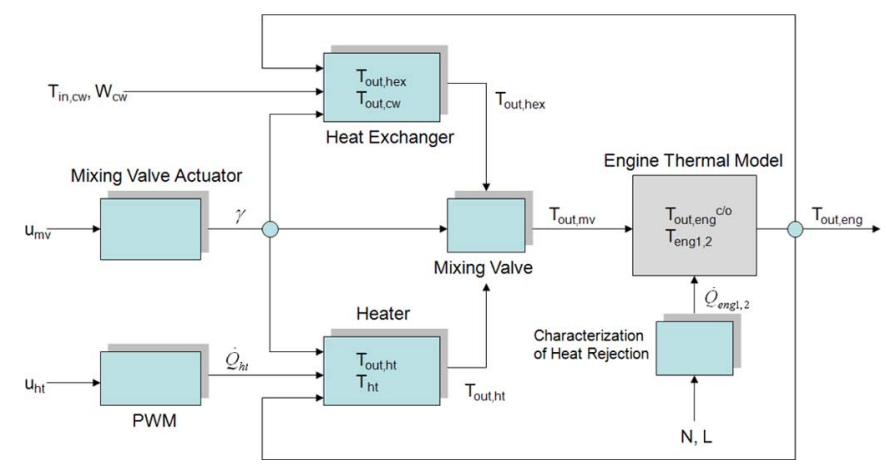

Fig. 2. Input/output system diagram.

acteristics are considered, and a recalibration procedure is outlined for mitigating performance degradation in the presence of these uncertainties.

Throughout the paper, the following nomenclature will be used to represent key variables, and whenever applicable, they will be used with proper subscript and superscript to denote the variable in a particular component and loop (for example, the $c$ and $o$ superscripts distinguish states belonging to the coolant and oil loops, respectively).

$m \quad$ Mass (kg).

$\dot{Q} \quad$ Rate of heat generation $(\mathrm{kW})$.

$W \quad$ Mass flow rate $(\mathrm{kg} / \mathrm{s})$.

$T$ Temperature $(C$ or $K)$.

$C \quad$ Specific heat $(\mathrm{kJ} / \mathrm{kg}-\mathrm{K})$.

$h A$ Convective heat transfer coefficient times interaction surface area $\left[(\mathrm{kW} / \mathrm{K})(\mathrm{s} / \mathrm{kg})^{0.8}\right]$.

$k A$ Conductive heat transfer coefficient times interaction surface area $(\mathrm{kW} / \mathrm{K})$.

It should be noted that while the hardware configuration and results of this paper are specific to an engine dynamometer application within a test cell, many of the component models presented in this paper may be used for modeling of in-vehicle cooling systems as well, with proper modifications (e.g., replacing the fluid-to-fluid heat exchanger used here with a fluid-to-air radiator present in a vehicle). Furthermore, the design methodology presented for this application has broad implications, particularly for overactuated systems with saturating actuators.

\section{Model DeVelopment And VALidation}

Fig. 2 shows the block diagram of the system represented in Fig. 1, where the states for each block are shown. The statespace model for each of these blocks is given in Section II-A. The shadowing on the mixing valve, heat exchanger, and heater blocks conveys the fact that two loops for coolant and oil exist in parallel, but pass through one (unshadowed) engine block. Note that each of these loops may be viewed as a subsystem consisting of two control inputs (mixing valve command, $u_{m v}$, and heater command, $u_{h t}$ ), four disturbance inputs (engine speed $N$, engine load $L$, cooling water temperature $T_{c w}$, and flow rate 
TABLE I

MODEL PARAMETERS AND STATES

\begin{tabular}{|c|c|c|}
\hline Subsystem & Parameters & States \\
\hline Heat Exchanger & $C_{\text {hot }}, C_{\text {cold }}, m_{\text {hot }}, m_{\text {cold }}, h A_{\text {hot }}, h A_{\text {cold }}, k A_{\text {hot }}, k A_{\text {cold }}$ & $T_{\text {out }, \text { hex }}, T_{\text {out }, c w}$ \\
Heater & $C_{\text {fluid }}, m_{\text {fluid }}, m C_{h t}, k A_{h t}, h A_{h t}$ & $T_{\text {out }, h t}, T_{h t}$ \\
Engine & $C^{c / o}, m^{c / o}, m C_{\text {eng } 1,2}, h A^{c / o}, k A^{c / o}, k A_{c p}, a_{j, i}$ & $T_{\text {out }, \text { eng }}^{c}, T_{\text {out }, \text { eng }}^{o}, T_{\text {eng } 1}, T_{\text {eng } 2}$ \\
\hline
\end{tabular}

TABLE II

CONTROL AND DISTURBANCE INPUTS

\begin{tabular}{|c|c|c|c|}
\hline Symbol & Input & Control/Disturbance & Range \\
\hline$\gamma$ & Bypass Ratio & Control & 0 to 1 \\
$\dot{Q}_{h t}$ & Heater Power & Control & 0 to $4.5 \mathrm{~kW}$ \\
$N$ & Engine Speed & Disturbance & 0 to $6000 \mathrm{rpm}$ \\
$L$ & Engine Load Torque & Disturbance & 0 to $200 \mathrm{~N}-\mathrm{m}$ \\
$T_{c w}$ & Cooling Water Temperature & Disturbance & 10 to 30 Degrees C \\
$W_{c w}$ & Cooling Water Flow Rate & Disturbance & 0 to $2 \mathrm{~kg} / \mathrm{s}$ \\
\hline
\end{tabular}

$W_{c w}$ ), and one performance output (temperature at engine outlet $T_{\text {out,eng }}$ ). The nominal values of $N, L, W_{c w}$, and $T_{c w}$ for that simulation or experiment will be referred to as the operating condition. The parameters, states, and inputs to the system are summarized in Tables I and II.

From the mixing valve command $u_{m v}$, we are able to control what we will refer to as the bypass ratio $\gamma$, where $\gamma=$ $W_{h t} / W$ is defined as the ratio of the flow through the heater to the total flow rate $(W)$. This bypass ratio, in conjunction with the system flow rate $W$, determines the flow rates through the heater and heat exchanger; therefore, $\gamma$ appears as an input to both the heater and heat exchanger blocks in Fig. 2. From the pulse-width-modulated (PWM) heater command $u_{h t}$, we may uniquely determine the power supplied by the heater $\dot{Q}_{h t}$ by a linear relationship. Finally, the heat inputs to the engine block $\dot{Q}_{\text {eng1,eng2 }}$ are determined by engine speed and load.

\section{A. Dynamic Model and Characterizing Equations}

For each loop, we consider the mixing valve, heater, and heat exchanger as subsystems. The engine, through which both loops circulate, is modeled as a heat source driven by engine speed and load, and the thermal inertia of the engine block is taken into account by the model. Without significant compromise of model accuracy, the following assumptions are made.

A1) both coolant and oil have constant density and specific heat;

A2) heaters and heat exchangers assume lumped parameters, with no energy storage in the heat exchanger "core";

A3) heater model assumes a single temperature state for the heater coil, allowing for energy storage in the coil;

A4) engine model assumes two states for engine block temperature, reflecting different temperature distribution, and heat rejection dynamics across the engine block;

A5) mixing valve assumes adiabatic and immediate mixing, resulting in a static relationship between the two inlet temperatures and the outlet temperature;

A6) heat losses in pipes are neglected.
1) Remark II.1: Assumption (A1) is valid throughout the operating window of the system, where the temperatures of the fluids are significantly less than their boiling points. Other assumptions have been shown to have negligible impact on the modeling results, except for (A5) and (A6), which will be elaborated on later, in Section II-C.

By conservation of energy, the inlet and outlet temperatures for the mixing valve are related by

$$
T_{\text {out }, m v}=\gamma T_{\text {out }, h t}+(1-\gamma) T_{\text {out }, \text { hex }} .
$$

The heat exchanger model contains temperature states for both the hot and cold flows. Heat is transferred across the heat exchanger "core" according to a logarithmic mean temperature difference law [12], yielding the following equations:

$$
\begin{aligned}
& \dot{T}_{\text {out }, \text { hex }}=\frac{1}{m_{\text {hot }}}\left((1-\gamma) W\left(T_{\text {out }, \text { eng }}-T_{\text {out }, \text { hex }}\right)-\frac{U A}{C_{\text {hot }}} \text { LMTD }\right) \\
& \dot{T}_{\text {out }, c w}=\frac{1}{m_{\text {cold }}}\left(W_{c w}\left(T_{\text {in }, c w}-T_{\text {out }, c w}\right)+\frac{U A}{C_{\text {cold }}} \text { LMTD }\right)
\end{aligned}
$$

where

- $U A$ is the overall heat transfer coefficient times the contact area-it is dependent on flow rate and may be expressed as

$$
U A=\frac{\left(h A_{\mathrm{hot}} W_{\mathrm{hot}}^{0.8}+k A_{\mathrm{hot}}\right)\left(h A_{\mathrm{cold}} W_{c w}^{0.8}+k A_{\mathrm{cold}}\right)}{h A_{\mathrm{hot}} W_{\mathrm{hot}}^{0.8}+k A_{\mathrm{hot}}+h A_{\mathrm{cold}} W_{c w}^{0.8}+k A_{\mathrm{cold}}}
$$

where $h A_{\text {hot/cold }}$ and $k A_{\text {hot/cold }}$ are parameters to be identified and $W_{\text {hot }}=(1-\gamma) W ; 1$

- LMTD is the logarithmic mean temperature difference between the hot and cold sides [12]. For a counterflow configuration, used in this system, it is defined as

$$
\text { LMTD }=\frac{\left(T_{\text {out }, \text { eng }}-T_{\text {out }, c w}\right)-\left(T_{\text {out,hex }}-T_{\text {in }, c w}\right)}{\ln \frac{T_{\text {out }, \text { eng }}-T_{\text {out }, c w}}{T_{\text {out }, \text { hex }}-T_{\text {in }, c w}}} .
$$

The heater model consists of a fluid temperature state and a coil temperature state, where

$$
\begin{aligned}
\dot{T}_{\text {out }, h t}= & \frac{1}{m_{\text {fluid }}} \\
& \times\left(\gamma W\left(T_{\text {out }, \text { eng }}-T_{\text {out }, h t}\right)\right. \\
& \left.\quad+\left(\frac{h A_{h t} W_{h t}^{8}+k A_{h t}}{C_{\text {fluid }}}\right)\left(T_{h t}-T_{\text {out }, h t}\right)\right) \\
\dot{T}_{h t}= & \frac{1}{m C_{h t}} \\
& \times\left(\dot{Q}_{h t}-\left(h A_{h t} W_{h t}^{8}+k A_{h t}\right)\left(T_{h t}-T_{\text {out }, h t}\right)\right) \\
W_{h t}= & \gamma W .
\end{aligned}
$$

In order to arrive at a representative engine model, two states are used to represent engine block temperatures, reflecting the different temperatures in the neighborhood of the coolant and

\footnotetext{
${ }^{1}$ The power of 0.8 in the expression for convective heat transfer arises from established empirical results [11].
} 


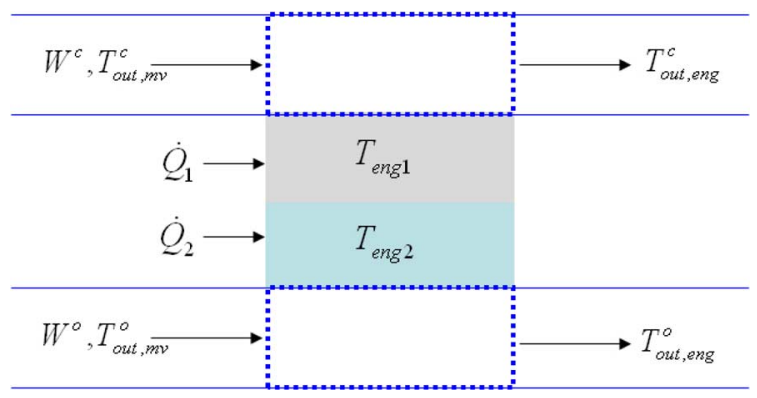

Fig. 3. Diagram for the engine subsystem model.

oil loops circulating through the engine block, as shown graphically in Fig. 3. This results in the fourth order engine subsystem model:

$$
\begin{aligned}
& \dot{T}_{\text {out }, \text { eng }}^{c}=\frac{1}{m^{c}} \\
& \left(W^{c}\left(T_{\text {out }, \text { eng }}^{c}-T_{\text {out }, m v}^{c}\right)\right. \\
& \left.+\left(\frac{h A^{c}\left(W^{c}\right)^{\cdot 8}+k A^{c}}{C^{c}}\right)\left(T_{\text {eng } 1}-T_{\text {out }, \text { eng }}^{c}\right)\right) \\
& \dot{T}_{\text {out,eng }}^{o}=\frac{1}{m^{o}} \\
& \times\left(W^{o}\left(T_{\text {out }, \text { eng }}^{o}-T_{\text {out }, m v}^{o}\right)\right. \\
& +\left(\frac{h A^{o}\left(W^{o}\right)^{\cdot 8}+k A^{o}}{C^{o}}\right) \\
& \left.\times\left(T_{\text {eng2 }}-T_{\text {out }, \text { eng }}^{o}\right)\right) \\
& \dot{T}_{\text {eng1 }}=\frac{1}{m C_{\text {eng1 }}} \\
& \times\left(\dot{Q}_{1}-\left(h A^{c}\left(W^{c}\right)^{.8}+k A^{c}\right)\left(T_{\text {eng1 }}-T_{\text {out,eng }}^{c}\right)\right. \\
& \left.+k A_{c p}\left(T_{\text {eng } 2}-T_{\text {eng } 1}\right)\right) \\
& \dot{T}_{\text {eng2 }}=\frac{1}{m C_{\text {eng2 }}}\left(\dot{Q}_{2}-\left(h A^{o}\left(W^{o}\right)^{8}+k A^{o}\right)\right. \\
& \times\left(T_{\text {eng } 2}-T_{\text {out,eng }}^{o}\right) \\
& \left.+k A_{c p}\left(T_{\text {eng } 1}-T_{\text {eng } 2}\right)\right)
\end{aligned}
$$

where $T_{\text {eng1,2 }}$ represent temperatures of the engine block states and $k A_{c p}$ represents the heat transfer within the engine itself, due to the temperature difference between the different regions of the engine block.

The heat rejection laws to the two portions of the engine block are modeled as functions of engine speed $(N)$ and load $(L)$

$$
\dot{Q}_{i}=a_{0, i}+a_{1, i} N+a_{2, i} L+a_{3, i} N L, \quad i=1,2
$$

where $a_{j, i}$ are parameters that reflect heat rejection characteristics for a given engine. The last term in (13) reflects heat rejection rate as a fraction of engine power $(N L)$. Other terms help to account for parasitic heat losses (through the first term) and for different efficiencies for different speed and load (through the second and third terms).

\section{B. Model Parameter Identification}

Parameters in the dynamic model (1)-(13) are identified experimentally. Temperature and flow rate measurements available for identification purposes are shown in Fig. 1. It should be noted that the fraction of flow passing through the heater and heat exchanger and the temperature of the heater coil or engine block were not measured. In order to identify the model parameters with available measurements, we manipulate the state equations for each component to derive a parametric model in order to use limited measurements. Here, we elaborate on the identification approach that was applied to each component.

1) Heat Exchanger Parameter Identification: For the heat exchanger, the availability of both $T_{\text {out,hex }}$ and $T_{\text {out, } c w}$ as measured variables leads to a simple two-step identification process in which we use steady-state data to identify the specific heats $C_{\text {hot/cold }}$, convection coefficients $h A_{\text {hot/cold }}$, and conduction coefficients $k A_{\text {hot } / \text { cold }}$, then use transient data to identify the fluid masses $m_{\text {hot/cold }}$. Even though the parameters do not appear linearly in the steady-state model [obtained by setting the right hand sides of (2) and (3) to 0], constrained optimization can be used to identify the parameters by minimizing steady-state modeling error. For this application, this constrained optimization was cast as a minimization of the cost function

$$
J\left(\hat{\theta}_{\mathrm{hex}}\right)=\sum_{i=1}^{N}\left(\epsilon_{i, \mathrm{hot}}^{2}+\epsilon_{i, \mathrm{cold}}^{2}\right)
$$

where

$$
\begin{aligned}
\epsilon_{i, \text { hot }} & =W_{\text {hot }}\left(T_{\text {out }, \text { eng }}-T_{\text {out }, \text { hex }}\right)-\frac{\hat{U A}}{\hat{C}_{\text {hot }}} \text { LMTD } \\
\epsilon_{i, \text { cold }} & =W_{c w}\left(T_{\text {in }, c w}-T_{\text {out }, c w}\right)+\frac{\hat{U A}}{\hat{C}_{\text {cold }}} \text { LMTD }
\end{aligned}
$$

and

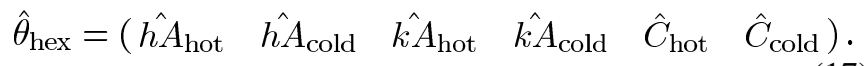

Once $\hat{\theta}_{\text {hex }}$ is identified, we proceed with the identification of the mass parameters $m_{\text {hot }}$ and $m_{\text {cold }}$ by substituting $\hat{\theta}_{\text {hex }}$ for $\theta_{\text {hex }}$ in (2) and (3) to obtain

$$
\begin{aligned}
& \dot{T}_{\text {out }, \text { hex }}=\frac{1}{m_{\text {hot }}}\left(W_{\text {hot }}\left(T_{\text {out }, \text { eng }}-T_{\text {out }, \text { hex }}\right)-\frac{\hat{U A}}{\hat{C}_{\text {hot }}} \text { LMTD }\right) \\
& \dot{T}_{\text {out }, c w}=\frac{1}{m_{\text {cold }}}\left(W_{c w}\left(T_{\text {in }, c w}-T_{\text {out }, c w}\right)+\frac{\hat{U A}}{\hat{C}_{\text {cold }}} \text { LMTD }\right)
\end{aligned}
$$

where the unknown parameters, $1 / m_{\text {hot }}$ and $1 / m_{\text {cold }}$ appear linearly. Therefore, a standard least squares algorithm can be used from this point forward to identify the unknown masses. To avoid using signal derivatives and reduce noise sensitivities, we apply a first-order filter to both sides of (18) and (19) before deriving the linear parametric model for identification, a procedure that is widely used and elaborated on in [10]. 
2) Heater Parameter Identification: For a given flow rate, the heater subsystem, with inputs $T_{\text {out,eng }}$ and $\dot{Q}_{h t}$ and output $T_{\text {out }, h t}$, may be modeled as a second-order linear system, taking $\gamma W$ as a parameter. This leads to a $2 \times 1$ transfer function matrix, which has the following properties.

1) The heater subsystem is linear and second order with respect to the inputs, $\dot{Q}_{h t}$ and $T_{\text {out,eng. }}$.

2) The transfer functions from $\left(\dot{Q}_{h t}\right)$ and $\left(T_{\text {out,eng }}\right)$ to $\left(T_{\text {out }, h t}\right)$ have relative degrees 2 and 1 , respectively.

3) The dc gain from the input $T_{\text {out,eng }}$ to the heater outlet temperature $T_{\text {out }, h t}$ is 1 .

Therefore, the heater can be represented by the input/output $(\mathrm{I} / \mathrm{O})$ relation

$$
Y(s)=\frac{b_{0}}{s^{2}+d_{1} s+d_{0}} U_{1}(s)+\frac{c_{1} s+d_{0}}{s^{2}+d_{1} s+d_{0}} U_{2}(s)
$$

where $Y$ is the outlet temperature, $T_{\text {out }, h t}$, and $U_{1}$ and $U_{2}$ represent $\dot{Q}_{h t}$ and $T_{\text {out,eng, }}$, respectively. Well-established methods [10] can be applied to identify $d_{0}, d_{1}, b_{0}$, and $c_{1}$.

The four transfer function coefficients are related to the physical heater model parameters by

$$
\begin{aligned}
d_{0} & =\frac{m_{\text {fluid }}\left(h A_{h t} W_{h t}^{8}+k A_{h t}\right)}{W_{h t} m C_{h t}} \\
d_{1} & =\frac{W_{h t}}{m_{\text {fluid }}}+\frac{h A_{h t} W_{h t}^{8}+k A_{h t}}{m C_{h t}}+\frac{h A_{h t} W_{h t}^{8}+k A_{h t}}{m_{\text {fluid }} C_{\text {fluid }}} \\
b_{0} & =\frac{m C_{h t}\left(h A_{h t} W^{\cdot 8}+k A_{h t}\right)}{m_{\text {fluid }} C_{\text {fluid }}} \\
c_{1} & =\frac{m_{\text {fluid }}}{W_{h t}} .
\end{aligned}
$$

In the linear heater model (21), the flow rate through the heater $W_{h t}$ is treated as a parameter that affects the transfer function coefficients. In order to use the linear model to identify heater parameters, identification was performed at various constant $W_{h t}$ (requiring a constant $W$ and $\gamma$ ), while engine load $L$ was varied in order to excite $T_{\text {out,eng }}$, thereby providing the needed excitation for parameter identification. By relying on data collected at different values of $W_{h t}$, it was possible to distinguish $h A_{h t}$ and $k A_{h t}$ (note that these parameters are indistinguishable through data collected at a single flow rate).

3) Engine Parameter Identification: The engine model represents the largest parameter identification challenge, since neither the engine block temperature states nor the heat input to the engine are known. However, the engine block temperature states $\left(T_{\text {eng1,2 }}\right)$ may be written in terms of the fluid temperature states $\left(T_{\text {out,eng }}^{c / o}\right)$ and heat rejection inputs, for steady-state data. This leads to a nonlinear parametric steady-state model from which the heat rejection coefficients, convection, and conduction coefficients,

$$
\hat{\theta}_{\mathrm{eng}, s s}=\left(\begin{array}{llll}
\hat{h A} & \hat{k A} & \hat{k A_{c p}} & \hat{a}_{j, i}
\end{array}\right)
$$

may be identified. Having identified these values, the engine subsystem, like the heater, may be expressed as a linear system, and a standard parameter identification algorithm may be used to determine

$$
\hat{\theta}_{\text {eng,trans }}=\left(\begin{array}{ll}
\hat{m}^{c / o} & \hat{m} C_{\text {eng } 1,2}
\end{array}\right) .
$$
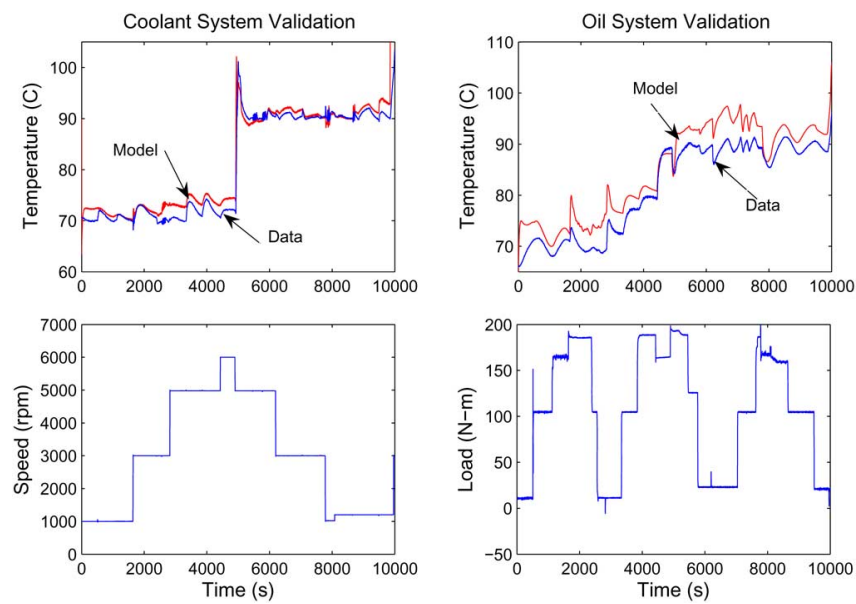

Fig. 4. Full system model validation results.

For a detailed treatment of this process, the reader is referred to the appendix.

\section{Model Validation}

The model validation results are shown in Fig. 4, where the predicted coolant and oil temperatures are compared with the measured data for a range of engine speeds and loads. The validation results in a root mean squared error (RMSE) of $2.4{ }^{\circ} \mathrm{C}$ and $4.4{ }^{\circ} \mathrm{C}$ for the coolant and oil loops, respectively.

In general, most model assumptions are valid based on the validation results. Validation traces do show that the model tends to overpredict the fluid temperatures, a result of neglecting heat losses through the piping in the system (A6). The error here is slightly more pronounced in the oil loop than in the coolant, due to the fact that the oil is flowing at a slower rate, and therefore losses to the ambient are a larger factor. Additionally, there is a very slight lag between the model prediction and the data, which is largely due to neglecting mixing valve dynamics (A5).

\section{DYNAMIC ANALYSIS}

Analysis of the validated model provides insights into the system dynamics that have led to an improved control design. Specifically, we will show, based on the model, that:

1) the coolant and oil loops have negligible interaction at typical operating conditions, and subsequent design and analysis will consider one loop at a time, with $T_{\text {out,eng }}$ held at setpoint for the other loop;

2) the two actuators possess very different dynamic control authorities in influencing $T_{\text {out,eng. The mixing valve is }}$ much faster to affect the outlet temperature than the heater, and control must take this into consideration.

The coupling of the two loops is shown in Fig. 5, where the Bode plots are presented for the transfer functions relating the engine inlet and outlet temperatures (a linear subsystem at constant flow rates), at typical flow rates of $5 \mathrm{~kg} / \mathrm{s}$ for coolant and $0.5 \mathrm{~kg} / \mathrm{s}$ for oil

$$
\left(\begin{array}{l}
T_{\text {out }}^{c} \\
T_{\text {out }}^{o}
\end{array}\right)=\left(\begin{array}{ll}
G_{11}(s) & G_{12}(s) \\
G_{21}(s) & G_{22}(s)
\end{array}\right)\left(\begin{array}{l}
T_{\text {in }}^{c} \\
T_{\text {in }}^{o}
\end{array}\right)
$$



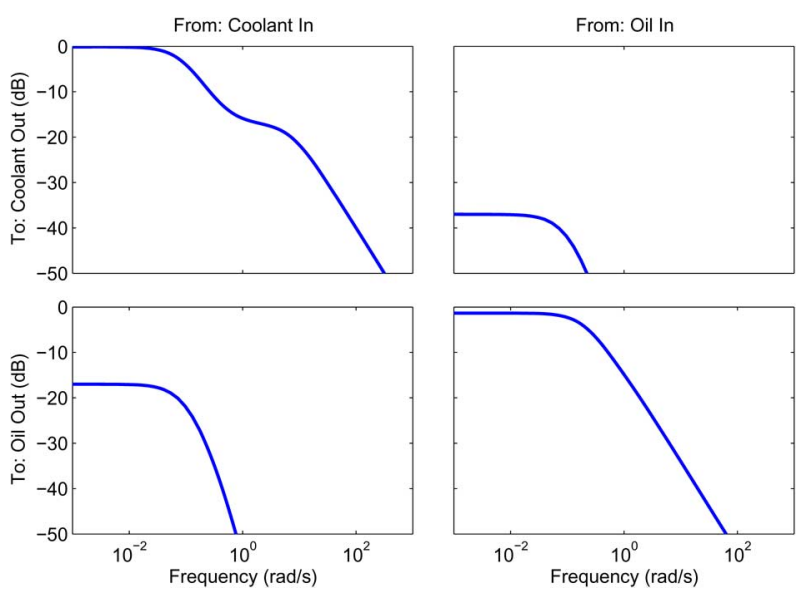

Fig. 5. Extent of loop decoupling.
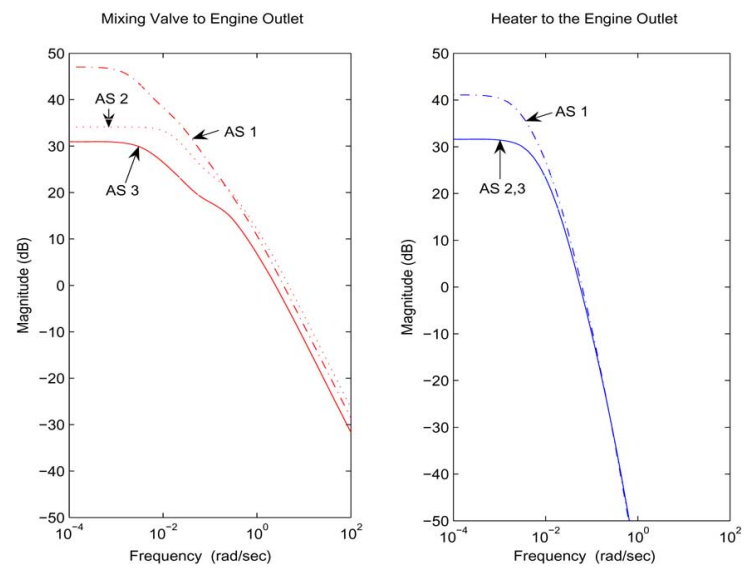

Fig. 6. Comparison of transfer functions between actuator inputs and outlet temperature at various actuator settings $\left(\mathrm{AS}^{\prime} s\right): \mathrm{AS} 1-\dot{Q}_{h t}=0 \mathrm{~kW}, \gamma=0.5$; $\mathrm{AS} 2-\dot{Q}_{h t}=4.5 \mathrm{~kW}, \gamma=0.5 ; \mathrm{AS} 3-\dot{Q}_{h t}=0 \mathrm{~kW}, \gamma=0.9$.

The relative magnitudes of the transfer functions on the diagonal are at least 5 times as large as the off-diagonal entries, indicating that one fluid has little thermodynamic influence on the other. Also, noteworthy is the fact that the oil temperature has less effect on the coolant than vice versa. This is because the coolant loop is the dominant loop in the system, occupying a much larger volume in the engine block than the oil loop. Similar results are obtained for different operating conditions.

With regard to the second analysis result, Fig. 6 shows, for the coolant loop, that the transfer function between the mixing valve input and engine outlet temperature has higher gain at high frequencies than that from the heater for all the actuator settings considered, suggesting that the valve possesses more transient control capability. The control inputs are normalized for the Bode plot in order to be comparable. The result for the oil loop exhibits the same trend. Therefore, to reserve sufficient transient control authority, it is critical to avoid operating the mixing valve near its saturation limit, and proper setting of the heater input may help to accomplish this goal. It is also noteworthy that the system linearizations vary widely depending on the actuator settings, and this variation should be taken into account when choosing where to operate the mixing valve.

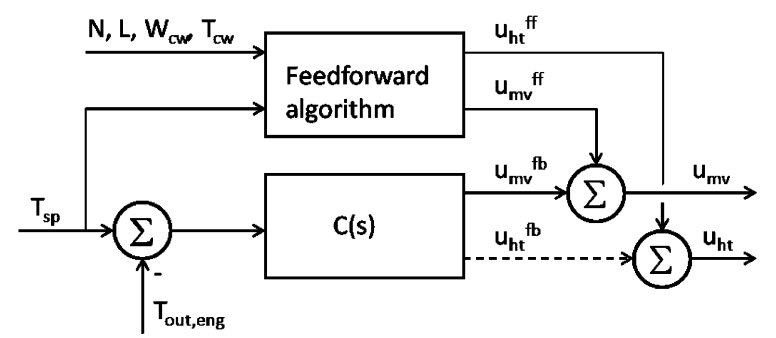

Fig. 7. Diagram of the complete control strategy.

\section{Control Design}

The problem of manipulating the engine outlet temperature has the following three important features which will be exploited in the control design:

1) overactuation, namely, two control inputs $u_{m v}$ and $u_{h t}$ for one performance variable;

2) different dynamic control authority for different actuators, as revealed by model analysis results presented in Fig. 6;

3) hard saturation limits on both actuators.

With overactuation, multiple combinations of control inputs will lead to the same steady-state output. Yet certain control inputs require the mixing valve (the "fast" control input) to be near its saturation limits, which reduces its effectiveness in influencing transient performance. Due to the nonlinearity in the system, choosing optimal control inputs does not amount to simply placing the valve in the middle of its operating range to avoid saturation. In this section, we propose a novel feedforward control strategy in which the dynamic authority of the system is evaluated about candidate control input combinations, based on the dynamic system model, and the combination that results in the greatest dynamic authority is selected as the feedforward control input $\left(u_{m v}^{f f}\right.$ and $\left.u_{h t}^{f f}\right)$. A relatively simple linear feedback control strategy is built on top of this (with outputs $u_{m v}^{f b}$ and $u_{h t}^{f b}$ ), with the overall control strategy depicted in Fig. 7.

\section{A. Feedforward Control Design}

Given that infinitely many different actuator settings can lead to the same steady-state engine outlet temperature, the objective of the static feedforward control design is to determine the optimal actuator setting for a given operating condition. ${ }^{2}$

A number of papers have considered the topic of overactuation in the presence of saturation constraints. In [13] and [14], control allocation methods are proposed for redundant robotic manipulators. In [15] and [16], different control allocation methods are compared for flight control applications. The common theme in all of these papers is that each of the actuators in the system affects the output in a similar way (i.e., all the actuators are dc motors for robots and control surfaces for flight control). To leverage transient authority of the different actuators, we extend the study of optimal control of overactuated systems by proposing a feedforward control methodology to deal simultaneously with saturation constraints, nonlinearities, and different dynamic authorities of the actuators.

\footnotetext{
${ }^{2}$ Actuator setting refers to any set of control inputs which achieve the desired setpoint at steady-state. Operating condition refers to the set of nominal values of the disturbance inputs to the system.
} 
In this work, the optimal actuator settings are determined by evaluating a single fitness function $F$ for each candidate actuator setting. Given that the objective of this controller is to track setpoints and reject disturbances, the total fitness $F$ is determined from setpoint tracking fitness $F_{r}$ and disturbance rejection fitness $F_{d}$

$$
F=F_{r} F_{d}^{\eta}
$$

where $\eta>0$ reflects the relative importance of setpoint tracking versus disturbance rejection. Here, we use a multiplicative, rather than additive, combination of $F_{r}$ and $F_{d}$ to avoid the potential problem of assigning a high total fitness to an actuator setting having high setpoint tracking fitness but essentially zero disturbance rejection fitness, or vice versa.

Our formulation for determining fitnesses assumes the following:

1) monotonic steady-state relationship between each input (control and disturbance) and output, i.e., no gain reversal;

2) system is bounded input, bounded output (BIBO) stable for each operating condition to be considered;

3) system is multi-input, single output (MISO).

Given that these assumptions are satisfied for the thermal management system, we define $F_{r}$ and $F_{d}$ as follows.

1) Definition IV.1 (Setpoint Tracking Fitness): Given an actuator setting $u_{0}$, operating condition $d_{0}$, and its corresponding steady-state output $y_{s s}\left(u_{0}, d_{0}\right)$, let $u^{+}$and $u^{-}$be the vector of maximum and minimum allowable control inputs, respectively. We then define

$$
\begin{aligned}
& \delta y_{u}^{+}(t) \triangleq y\left(u^{+}, t\right)-y_{s s}\left(u_{0}, d_{0}\right) \\
& \delta y_{u}^{-}(t) \triangleq-y\left(u^{-}, t\right)+y_{s s}\left(u_{0}, d_{0}\right)
\end{aligned}
$$

where $y\left(u^{+}, t\right)$ and $y\left(u^{-}, t\right)$ are the open-loop responses to step inputs from $u_{0}$ to $u^{+}$and $u^{-}$, respectively. The setpoint tracking fitness $F_{r}$ is defined by

$$
F_{r} \triangleq \min \left\{\int_{0}^{T} w(t) \delta y_{u}^{-}(t) d t, \int_{0}^{T} w(t) \delta y_{u}^{+}(t) d t\right\}
$$

where $w(t)>0$ is a weighting function.

The fitness function defined in (31) represents the capability of the system, around a particular actuator setting and operating condition, to influence the output (and therefore track setpoints) under the saturation constraints of the actuators. The weighting function $w(t)$ is used to place emphasis on the transient response. In this paper, we use

$$
w(t)=e^{-t / \tau}
$$

which reflects the importance of a fast initial response.

For disturbance rejection fitness $F_{d}$ recognizing the fact that the capability of disturbance rejection is favorable when the actuators have high authority in influencing the output and the disturbances have low authority in influencing the output, we give the following definition for disturbance rejection fitness.

2) Definition IV.2 (Disturbance Rejection Fitness): Given $u_{0}, d_{0}$, and the corresponding steady-state output $y_{s s}\left(u_{0}, d_{0}\right)$,

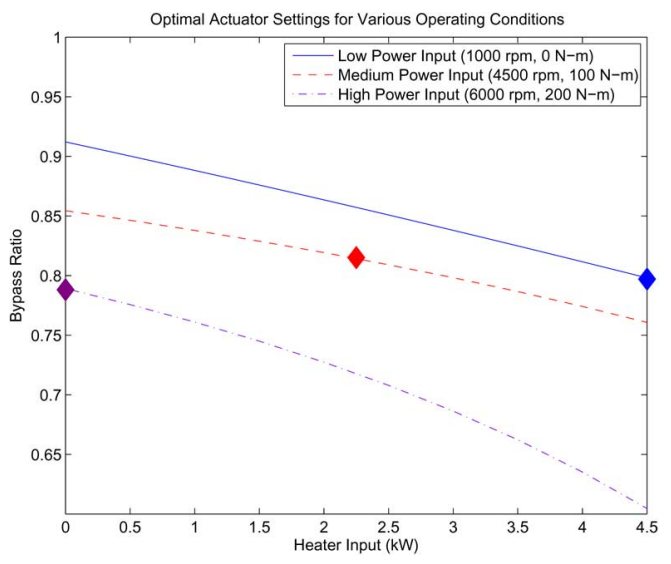

Fig. 8. Comparison of feasible and optimal (diamonds) actuator settings for various operating conditions.

let $d^{+}$and $d^{-}$be the vector of unit step disturbances to the system, in the direction of increasing and decreasing output, respectively. We then define

$$
\begin{aligned}
& \delta y_{d}^{+}(t) \triangleq y\left(d^{+}, t\right)-y_{s s}\left(u_{0}, d_{0}\right) \\
& \delta y_{d}^{-}(t) \triangleq-y\left(d^{-}, t\right)+y_{s s}\left(u_{0}, d_{0}\right)
\end{aligned}
$$

where $y\left(d^{+}, t\right)$ and $y\left(d^{-}, t\right)$ are the open-loop responses to step inputs from $d_{0}$ to $d^{+}$and $d^{-}$, respectively. The disturbance rejection fitness $F_{d}$ is defined by

$$
F_{d} \triangleq \min \left\{\frac{\int_{0}^{T} w(t) \delta y_{u}^{-}(t) d t}{\int_{0}^{T} w(t) \delta y_{d}^{+}(t) d t}, \frac{\int_{0}^{T} w(t) \delta y_{u}^{+}(t) d t}{\int_{0}^{T} w(t) \delta y_{d}^{-}(t) d t}\right\}
$$

where $w(t)$ is a weighting function, and $y_{u}^{+}$and $y_{u}^{-}$are defined in Definition 4.1.

Qualitatively, the numerator in $F_{d}$ captures the same system properties as $F_{r}$, namely the ability of the actuators to impart changes to the system output, whereas the denominator captures the ability of the disturbances to impart changes to the output.

Using the proposed fitness function, the resulting optimal actuator settings are highlighted in Fig. 8 (optimal actuator settings are denoted by diamonds) for various speed and load conditions of the coolant loop, when the temperature setpoint is $90^{\circ} \mathrm{C}$, with the cooling water at a temperature of $20^{\circ} \mathrm{C}$ and flow rate of $0.4 \mathrm{~kg} / \mathrm{s}$. In this case, we choose $\eta=1$ and $\tau=30 \mathrm{~s}$ in (28) and (32). Consistent with intuition, the proposed fitness function suggests that it is advantageous to have the heater on at low power, off at high power, and at an intermediate PWM state somewhere in between. The proposed fitness function provides a metric to quantify the optimal transition point from having the heater fully on to having it fully off. This results in a strategy where the mixing valve (the fast actuator) is kept away from its saturation limits $(\gamma=0.05$ and $\gamma=0.95)$. However, note that in all three cases, the optimal mixing valve position is not precisely at the middle $(\gamma=0.5)$ of its operating range. Instead, the optimization takes the system nonlinearity into account by evaluating the proposed fitness function. 


\section{B. Implementation of the Feedforward Control Design Via Support Vector Classification}

The optimization described in the previous section must be carried out offline, since the requirement of simulating the system far exceeds our online computational capability. Therefore, an efficient and accurate means of storing and retrieving optimal actuator settings is required for the online determination of the feedforward control input, $u^{f f}$. This could be done with a four-dimensional lookup table with inputs of $N, L, T_{\mathrm{in}, c w}$, and $W_{c w}$ (and an additional dimension for $T_{s p}$ if setpoint variations are large enough) and outputs of $u_{m v}^{f f}$ and $u_{h t}^{f f}$. With this method, a very coarse lookup table with only three values for each variable amounts to 81 actuator settings that need to be maintained.

However, note that there exists a large set of operating conditions in which the heater is optimally either fully on or fully off, and a relatively small range in which the optimal actuator setting is somewhere in between. We propose a support vector machine (SVM) classification to improve the efficiency of the feedforward control implementation while preserving the accuracy.

In order to perform the classification, we cast each operating condition as a four-dimensional vector $d_{0}$, where

$$
d_{0}=\left(\begin{array}{llll}
N & L & T_{\text {in }, c w} & W_{c w}
\end{array}\right) .
$$

A set of $n$ ( $n=500$ in our case) training operating conditions $d_{t r, i}, i=1, \ldots, n$, is generated, and the corresponding optimal actuator settings are calculated (using the dynamic system model to calculate fitnesses) and assigned to one of the following three sets:

\section{S0 heater is optimally off;}

$S 1$ heater is optimally on;

$S 2$ remaining operating conditions.

To derive an SVM classification that effectively sorts operating conditions into these sets, we use the following $\mathrm{RBF}$ kernel:

$$
K\left(d_{0}, d_{i, t r}\right)=e^{-\left\|d_{0}-d_{t r, i}\right\|^{2} / \sigma^{2}} .
$$

and classifier function

$$
f\left(d_{0}\right)=\sum_{i=1}^{n}\left(w_{i} K\left(d_{0}, d_{t r, i}\right)\right)
$$

Here, $\sigma$ is a tuning parameter, and $w_{i}$ represent weights applied to the points selected as support vectors, as determined by the SVM algorithm. For this application, DTREG software [18] (which includes software for decision trees, support vector machines, and logistic regression) is used to compute the support vectors and corresponding $w_{i}$. All of the training data belonging to $S 0$ or $S 1$ were used as inputs, and the software selected 40 of these training points as support vectors, leading to 40 non-zero $w_{i}$ whose signs depend on which set $(S 0$ or $S 1$ ) the corresponding training data point $d_{t r, i}$ belongs to.
TABLE III

SUMMARY OF BENEFITS OF SUPPORT VECTOR CLASSIFICATION

\begin{tabular}{|c|c|c|}
\hline Method & No. Stored Vars. & Accuracy (percent) \\
\hline SVM & 204 & 98 \\
$3^{4}$-cell Table & 170 & 80 \\
$6^{4}$-cell Table & 2612 & 90 \\
$10^{4}$-cell Table & 20036 & 97 \\
\hline
\end{tabular}

Once this classification is performed, the feedforward control law for the heater is implemented as

$$
u_{h t}^{f f}\left(d_{0}\right)= \begin{cases}0, & f\left(d_{0}\right)<f_{l b} \\ u_{h t}^{\max }, & f\left(d_{0}\right)>f_{u b} \\ u_{h t}^{\max } \frac{f\left(d_{0}\right)-f_{l b}}{f_{u b}-f_{l b}}, & f_{l b}<f\left(d_{0}\right)<f_{u b}\end{cases}
$$

where $f_{l b}$ and $f_{u b}$ are thresholds that minimize the number of misclassified training points.

Once the heater input has been set, a unique bypass ratio $\gamma$ can be determined that will achieve the desired setpoint at steady state.

The support vector algorithm used here provides a better combination of accuracy and computational simplicity than a lookup table does, as summarized in Table III. Here, we compare the use of the SVM method with the alternative method of gridding the space of $d_{0}$, then basing actuator settings on whichever cell in the grid $d_{0}$ happens to belong to. Note that we are counting the number of stored scalar variables in the middle column (for example, an actuator setting, having both a heater and valve component, counts as two). Accuracy is computed as the percent of random validation points that are classified correctly. A validation point is said to be correctly classified if the computed actuator setting (based on whichever method is being considered) falls into the same class $(S 0, S 1$, or $S 2)$ as the optimal actuator setting. As with the training data, the optimal actuator settings for all of the validation data points are computed using the model.

\section{Feedback Control Design}

Having shown the procedure for designing and implementing $u_{m v}^{f f}$ and $u_{h t}^{f f}$, we now turn to $u_{m v}^{f b}$ and $u_{h t}^{f b}$. Because the mixing valve possesses significantly greater dynamic authority than the heater, it serves as the primary mechanism for feedback. In fact, the role of the heater in feedback is so minimal, as we shall later justify in simulations, that with the appropriate value of $u_{h t}^{f f}$, we can take $u_{h t}^{f b}=0$. A simple, but effective, feedback law for the mixing valve was designed in the following manner:

1) initially, a full-order, LQG control design was performed, resulting in a full-order compensator $C_{\text {full }}(s)$, following [17];

2) examination of $C_{\text {full }}(s)$ showed that $C_{\text {full }}(s)$ could be approximated reasonably closely using a simple PI controller, yielding a simple and manageable feedback control law for implementation in the test cell;

3) filter was augmented to this resulting PI controller in order to reduce the gain of the controller at high frequencies 

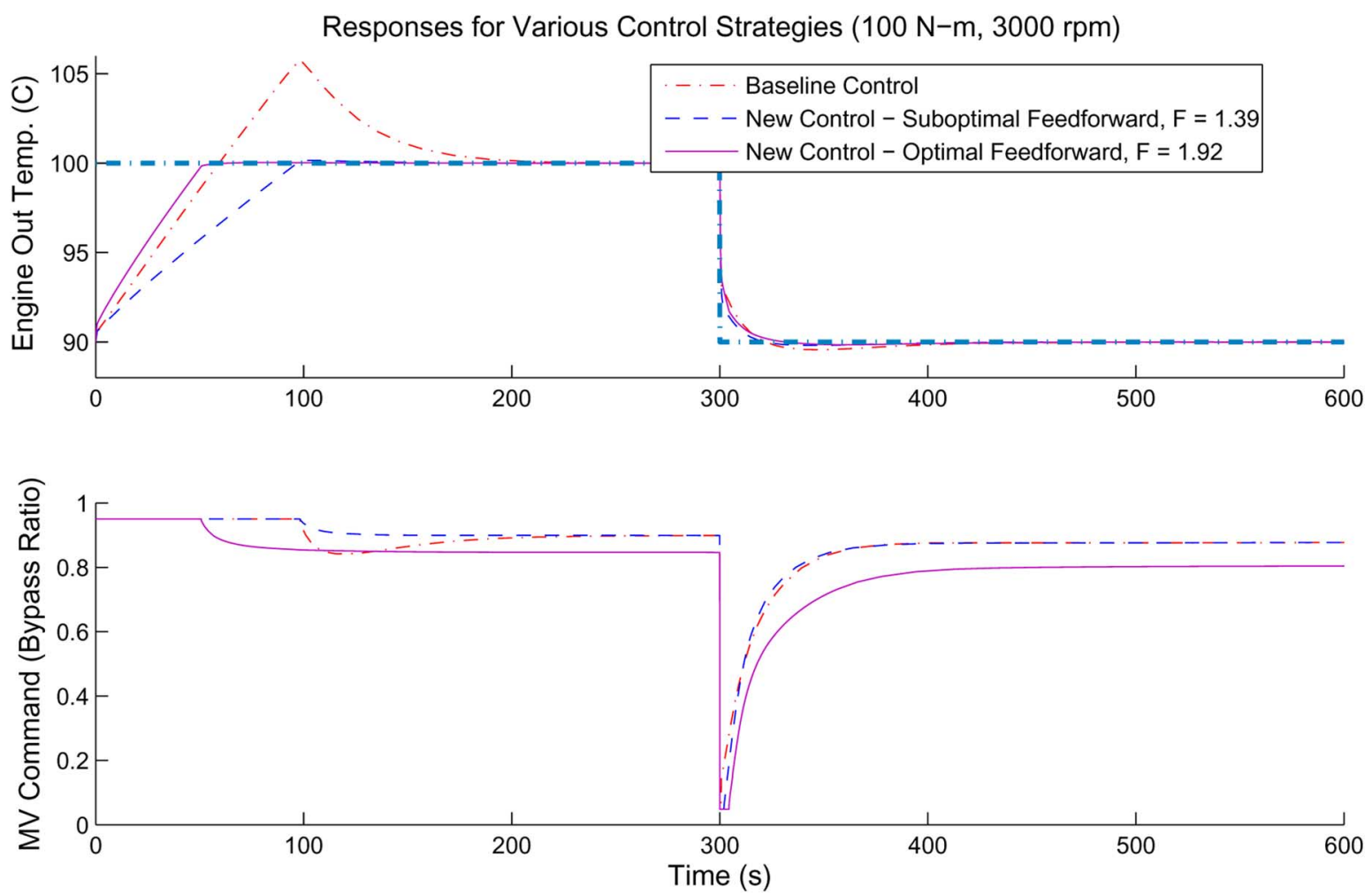

Fig. 9. Closed-loop simulation results for a $10{ }^{\circ} \mathrm{C}$ setpoint change (from $90{ }^{\circ} \mathrm{C}$ to $100{ }^{\circ} \mathrm{C}$ ) with only the mixing valve for feedback.

(making the closed-loop system more robust to delays and unmodeled dynamics), while maintaining high gain at middle and low frequencies.

The final feedback controller for this system is a simple PI controller + lag filter of the form

$$
C(s)=\frac{K_{p} s+K_{i}}{s} \frac{a s+1}{b s+1} .
$$

1) Remark IV.1: Since the proposed control strategy will, in general, use both actuators simultaneously, it is theoretically possible to encounter a situation in which $\gamma=0$ (routing all flow through the heat exchanger) and $\dot{Q}_{h t}>0$. For this system, since none of the optimal actuator settings are at or near $\gamma=0$, this scenario is not likely to happen for a sustained period of time. However, a safeguard strategy can be put in place to deal with transient events in which this failure scenario is detected. In our case, a lower bound of $\gamma=0.05$ is imposed. In addition, a flow switch exists in the hardware, which shuts the heater off when minimal flow through the heater circuit is detected.

\section{SimUlation AND EXPERIMENTAL VALIDATION}

With a combined feedforward/feedback controller in place, the performance of the system is evaluated using both modelbased simulation and experimental validation. This new control strategy is compared to the baseline control strategy, which utilizes only one actuator at a time, using the mixing valve for decreasing $T_{\text {out,eng }}$ and the heater for increasing $T_{\text {out,eng }}$. Since the baseline controller is a proprietary product based on an off-the-shelf PI control scheme, not all of its features can be replicated in simulation. However, its basic structure is as follows:

$$
\begin{aligned}
& u_{\text {total }}(t)= k_{p}\left(T_{s p}(t)-T_{\text {out }, \text { eng }}(t)\right) \\
&+k_{i} \int_{0}^{t}\left(T_{s p}(\tau)-T_{\text {out }, \text { eng }}(\tau)\right) d \tau \\
& \gamma(t)= \begin{cases}\gamma_{\max }, & u_{\text {total }}(t) \geq 0 \\
\gamma_{\max }+u_{\text {total }}(t), & u_{\text {total }}(t)<0\end{cases} \\
& \dot{Q}_{h t}(t)= \begin{cases}0, & u_{\text {total }}(t) \leq 0 \\
u_{\text {total }}(t), & u_{\text {total }}(t)>0 .\end{cases}
\end{aligned}
$$

This controller will be used in our simulation study to provide a benchmark.

Fig. 9 shows the numerical simulation results of the closedloop system with different feedforward actuator settings. Here, the impact of the proper choice of feedforward actuator setting on transient system performance is illustrated in a medium power setting, where it is optimal to have the heater fully on $(4.5 \mathrm{~kW})$. In Fig. 9, we see that actuator setting can greatly affect the ability to track changing setpoints. With the new control strategy and the optimal actuator setting, the 10 degree temperature rise occurs faster than with the baseline strategy. Additionally, because the new strategy relies on the mixing valve as the primary feedback mechanism, it results in faster settling of transients after the setpoint is reached, whereas the baseline strategy switches between using the heater (with large thermal inertia) and the valve, and results in significant transients.

Because our implemented control strategy relies on the valve alone for feedback control $\left(u_{h t}^{f b}=0\right)$, which has proven effective for appropriate actuator setting selection, it is worth comparing responses when the heater is used in the feedback loop as 

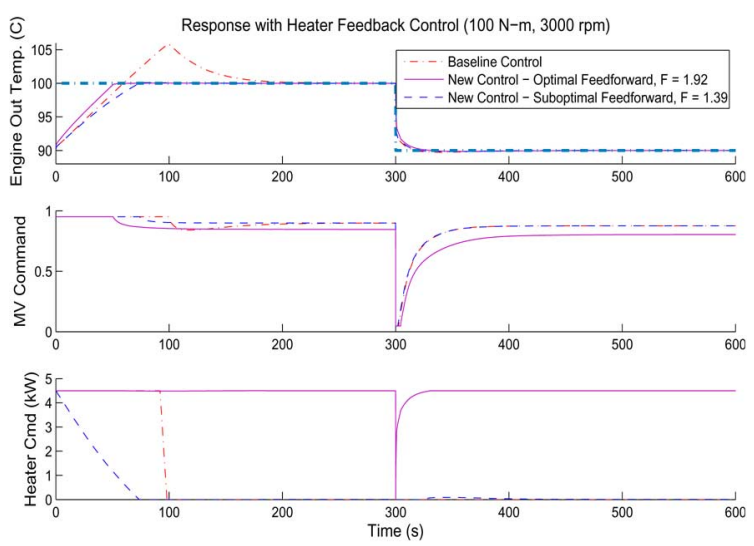

Fig. 10. Closed-loop simulation results for a $10{ }^{\circ} \mathrm{C}$ setpoint change (from $90^{\circ} \mathrm{C}$ to $100^{\circ} \mathrm{C}$ ) with both the valve and heater active in the feedback loop.

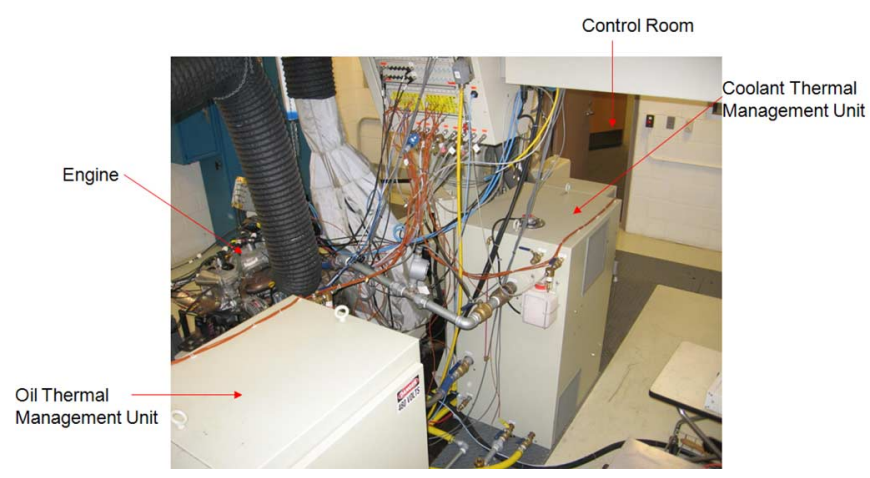

Fig. 11. Engine test cell setup.

well. Fig. 10 shows that use of the heater for feedback does improve the response for suboptimal feedforward actuator settings but not significantly when the feedforward is selected optimally according to the proposed fitness function of Section IV. Plots presented in this section all refer to the coolant loop, but the trends in oil loop performance are the same.

The control strategy was also tested experimentally. The experimental setup consisted of both the oil and coolant loops set up in an engine test cell, along with the engine and dynamometer. The control algorithm was implemented as a MATLAB function that was called by the test cell's data acquisition system (Atlas), which handles the inputs (thermocouple voltage inputs) and outputs (current supplied to the heater and valve). A photograph of this experimental setup is shown in Fig. 11.

Experimental results show a significant advantage in using the new control design over the baseline, off-the-shelf controller. Fig. 12 shows the RMS error between the temperature and setpoint as well as the percentage of time spent outside control specifications for both the new control design and the baseline, over a wide range of testing conditions.

1) Remark V.1: For this application, RMSE and time spent outside control specifications are our only two performance metrics, which the new control methodology succeeds in improving upon. However, the new control methodology does result in a larger amount of power expended by the heater, which represents a tradeoff that may need to be made in other

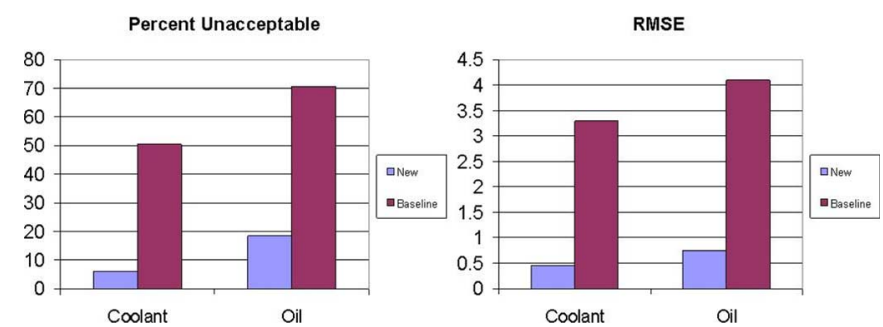

Fig. 12. Performance comparison of new and baseline controllers, as measured by RMSE and percent of time spent outside of control specifications (two original metrics for assessing control quality).
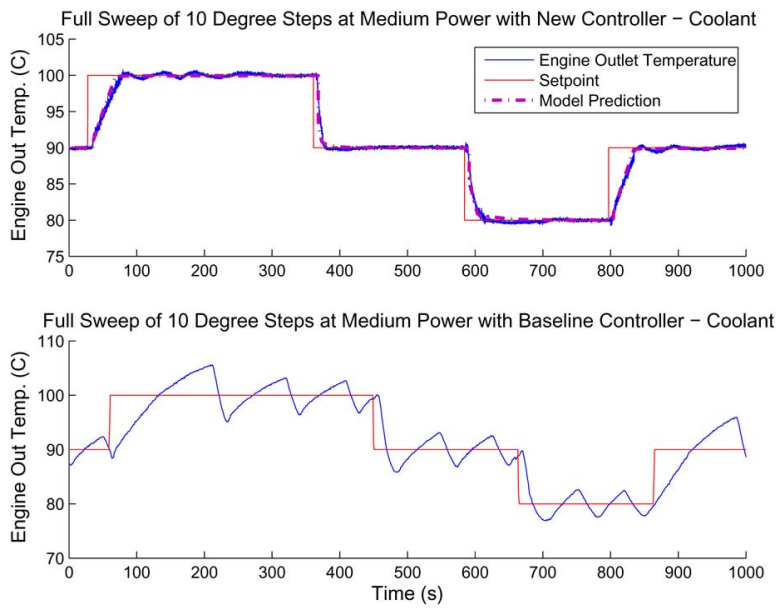

Fig. 13. Dynamometer results for a sweep of step inputs at medium power-new controller.

thermal management applications where energy conservation is paramount.

Fig. 13 shows the time traces for a sequence of step inputs at a $3000 \mathrm{rpm}, 100 \mathrm{~N} \cdot \mathrm{m}$ operating condition for the new control system and baseline control system, respectively, demonstrating that the new controller produces better results over a wide range of testing conditions. Additionally, the model prediction in Fig. 13 matches the actual response closely for the new control strategy, illustrating the fidelity of the model for use in control design and analysis.

\section{Performance Sensitivity and Recalibration PROCEDURE}

In order for the thermal management system to be effective in a testing environment in which many different engines may be tested on the same dynamometer, the controller design must either be robust to variations in engine parameters or be easily recalibrated when a change is made.

In this section, we consider the following three main sets of engine parameters:

1) heat rejection coefficients;

2) heat transfer coefficients;

3) engine block and fluid masses.

Consider the case when an existing engine is replaced with a less efficient one, which rejects more heat to coolant and oil. If the variation in engines is large enough, there will exist operating conditions where the optimal actuator settings for the new engine will be exactly the opposite of the optimal settings for 

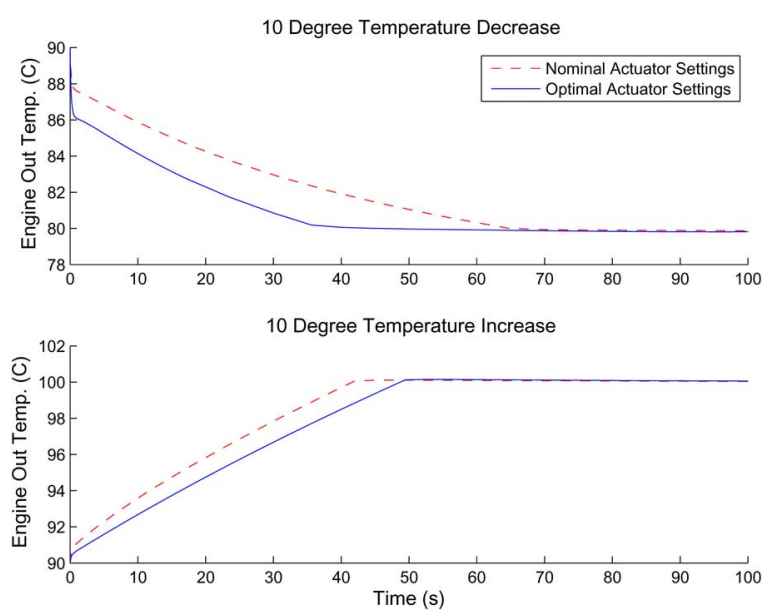

Fig. 14. Optimal versus nominal control for a -10 degree setpoint change with higher than expected heat rejection.

the original engine. The consequences of this parameter variation are shown in Fig. 14, where "nominal actuator settings" and "optimal actuator settings" refer to the controller designed using the original heat rejection coefficients and the adjusted coefficients, respectively. The need to recalibrate the feedforward control is demonstrated in Fig. 14, where reoptimizing yields a favorable tradeoff between temperature decrease and temperature increase capability.

On the other hand, with $\pm 20 \%$ variation in heat transfer coefficients or masses (engine block and fluid), the closed-loop performance is essentially unchanged with recalibration. In all cases, the feedforward commands, remain exactly the same, whereas the feedback gains vary by less than $5 \%$.

1) Remark VI.1: Through simulation, we have demonstrated the benefits of recalibrating the feedforward control when an engine change is made. However, recalibrating the full feedforward map for a different engine could result in a tedious data collection and optimization task. To simplify this task, we note that the system model with $N$ and $L$ as inputs is dependent on the heat rejection coefficients $\left(a_{i}\right)$, the model with $\dot{Q}$ and $W$ as inputs is not. This fact allows us to recast the feedforward control as a function of $\dot{Q}$ and $W$, which is independent of the engine, after which the recalibration can be performed by identifying the heat rejection coefficients $a_{i}$.

To recalibrate the heat rejection model, we treat the closedloop system, with $\dot{Q}$ and $W$ as inputs, as invariant for a given $\gamma$ and $\dot{Q}_{h t}$. In this case, for small perturbations in speed and load, the closed-loop system may be represented as a linear parametric model for $a_{1}, a_{2}$, and $a_{3}$

$$
\begin{aligned}
T_{\text {out }, \text { eng }}(s)+D(s) W(s)= & C(s) \Delta \dot{Q}(s), \\
\Delta \dot{Q}(s)= & a_{1} \Delta N(s)+a_{2} \Delta L(s) \\
& +a_{3} \Delta(N L)(s) .
\end{aligned}
$$

The model facilitates the identification of $a_{1}, a_{2}$, and $a_{3}$ using a recursive least squares algorithm. For this recalibration, it is assumed that the change in $a_{0}$ under a change in engines is minimal.

\section{CONCLUSION AND FUTURE WORK}

In this paper, we have proposed and validated a dynamic model for a thermal management system that has guided a model-based control design. This design has proven to yield significant performance advantages over the baseline. In particular, we have explored the area of overactuated systems in the presence of hard saturation limits and different dynamic actuator authorities, and have proposed a novel method to design the feedforward controller. Further research and development will focus on optimal control of overactuated systems, as well as extending the proposed design framework to more general cases.

\section{APPENDIX}

A two-step engine parameter identification procedure will be described in this appendix, to complement the discussion in Section II. First, steady-state data is used to identify heat transfer parameters and the coefficients for the laws governing heat rejection from the engine $(\dot{Q})$. Then transient data is used to identify mass parameters.

Recalling the notation in Section II, we cast the steady-state and transient parameters in vectors $\theta_{\mathrm{eng}, s s}$ and $\theta_{\mathrm{eng}, \text { trans }}$

$$
\theta_{\text {eng,ss }}=\left(\begin{array}{llll}
h A^{c / o} & k A^{c / o} & k A_{c p} & a_{i, j}
\end{array}\right)
$$

and

$$
\theta_{\text {eng,trans }}=\left(\begin{array}{ll}
m^{c / o} & m C_{\text {eng } 1,2}
\end{array}\right) .
$$

At steady-state, the equations associated with the engine subsystem become

$$
\begin{aligned}
0= & W^{c}\left(T_{\text {out }, \text { eng }}^{c}-T_{\text {out }, m v}^{c}\right) \\
& +\left(\frac{h A^{c}\left(W^{c}\right)^{8}+k A^{c}}{C^{c}}\right)\left(T_{\text {eng } 1}-T_{\text {out }, \text { eng }}^{c}\right) \\
0= & W^{o}\left(T_{\text {out }, \text { eng }}^{o}-T_{\text {out }, m v}^{o}\right) \\
& +\left(\frac{h A^{o}\left(W^{o}\right)^{\cdot 8}+k A^{o}}{C^{o}}\right)\left(T_{\text {eng } 2}-T_{\text {out }, \text { eng }}^{o}\right) \\
0= & \dot{Q}_{1}-\left(h A^{c}\left(W^{c}\right)^{8}+k A^{c}\right)\left(T_{\text {eng } 1}-T_{\text {out }, \text { eng }}^{c}\right) \\
& +k A_{c p}\left(T_{\text {eng } 2}-T_{\text {eng } 1}\right) \\
0= & \dot{Q}_{2}-\left(h A^{o}\left(W^{o}\right)^{8}+k A^{o}\right)\left(T_{\text {eng } 2}-T_{\text {out }, \text { eng }}^{o}\right) \\
& +k A_{c p}\left(T_{\text {eng } 1}-T_{\text {eng } 2}\right) .
\end{aligned}
$$

Using (50) and (51), we may express the engine states $T_{\text {eng1 }}$ and $T_{\text {eng2 }}$ in terms of the measured states and $\theta_{s s}$ as

$$
\left(\begin{array}{c}
T_{\text {eng1 }} \\
T_{\text {eng2 }}
\end{array}\right)=\left(\begin{array}{cc}
b_{1}+b_{2} & -b_{1} \\
-b_{1} & b_{1}+b_{3}
\end{array}\right)^{-1}\left(\begin{array}{l}
\dot{Q}_{1}+b_{2} T_{\text {out,eng }}^{c} \\
\dot{Q}_{2}+b_{3} T_{\text {out }, \text { eng }}^{o}
\end{array}\right)
$$

where

$$
\begin{aligned}
& b_{1}=k A_{c p} \\
& b_{2}=k A^{c}+h A^{c}\left(W^{c}\right)^{\cdot 8} \\
& b_{3}=k A^{o}+h A^{o}\left(W^{o}\right)^{\cdot 8} .
\end{aligned}
$$

Substituting (52) into (48) and (49), we have 2 steady-state equations that relate $\dot{Q}_{1}, \dot{Q}_{2}$, and $T_{\text {out, } m v}^{c / o}$ to $T_{\text {eng,out }}^{c / o}$ through 13 parameters $(5$ heat transfer parameters and 8 heat rejection 
parameters). The steady-state equations do not lead to a linear parametric model. As we did for the heat exchanger validation, offline optimization was used to identify unknown parameters.

Having identified the coefficients to the heat rejection law, we were able to estimate $\dot{Q}$ in terms of engine speed and load. The availability of this input and the knowledge of system flow rates $(W)$ allows us to treat the engine subsystem as a linear system, with $W^{c / o}$ taken as parameters, where the transient parameters contained in $\hat{\theta}_{\text {trans }}$ may be identified.

This linear engine subsystem model may be represented in the form of a $4 \times 2$ transfer function matrix, partitioned as

$$
\left(\begin{array}{c}
Y_{1} \\
Y_{2}
\end{array}\right)=\left(G\left(\theta_{s s, \text { eng }}, \theta_{\text {trans,eng }}\right)\right)\left(\begin{array}{c}
U_{1} \\
U_{2} \\
U_{3} \\
U_{4}
\end{array}\right)
$$

where $Y_{1}$ and $Y_{2}$ represent the coolant and oil engine outlet temperatures, respectively, $G$ is a $4 \times$ transfer function matrix, $U_{1}$ and $U_{2}$ represent the coolant and oil engine inlet temperatures, and $U_{3}$ and $U_{4}$ represent $\dot{Q}_{1,2}$, calculated according to

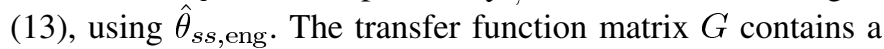
total of 24 coefficients which may be identified using a standard least squares technique. We cast these coefficients into a vector $c$. Each element in $c$ is related to the original physical parameters in $\theta_{\text {trans,eng }}$ by known but nonlinear relations. Note that $c$ has dimension of 24 while $\theta_{\text {trans,eng }}$ has a dimension of 4 . Therefore, there is no one-to-one mapping between $c$ and $\theta_{\text {trans,eng }}$ from which $\theta_{\text {trans,eng }}$ can be determined. For our application, $\theta_{\text {trans,eng }}$ is determined by minimizing

$$
J=\left\|c-c\left(\hat{\theta}_{\text {trans,eng }}\right)\right\|
$$

where $c$ is the vector resulting from applying standard least squares identification to (56).

\section{REFERENCES}

[1] P. Setlur, J. Wagner, D. Dawson, and J. Chen, "Nonlinear controller for automotive thermal management systems," in Proc. Amer. Control Conf., 2003, pp. 4699-4704.

[2] H. Couetouse and D. Gentile, "Cooling system control in automotive engines," SAE, Warrendale, PA, 1992.

[3] J. Wagner, I. Paradis, E. Marotta, and D. Dawson, "Enhanced automotive engine cooling systems-A mechatronics approach," Int. J. Veh. Des., vol. 28, pp. 1-3, 2002.

[4] J. Wagner, M. Ghone, D. Dawson, and E. Marotta, "Coolant flow control strategies for automotive thermal management systems," SAE, Warrendale, PA, 2002-01-0713, 2002.

[5] P. Setlur, J. Wagner, D. Dawson, and E. Marotta, "An advanced engine thermal management system: Nonlinear control and test," IEEE/ASME Trans. Mechatronics, vol. 10, no. 2, pp. 210-220, Apr. 2005.

[6] X. Zou, J. A. Jordan, and M. Shillor, "A dynamic model for a thermostat," J. Eng. Math., vol. 36, pp. 291-310, 1999.

[7] G. Einaudi and W. Mortara, "Engine cooling electronic control system," SAE, Warrendale, PA, 885085, 1988.

[8] A. Kenny, C. Bradshaw, and B. Creed, "Electronic thermostat system for automotive engines," SAE, Warrendale, PA, 880265, 1988.

[9] C. Vermillion, J. Sun, K. Butts, and A. Hall, "Modeling and analysis of a thermal management system for engine calibration," in Proc. IEEE Conf. Control Appl., 2006, pp. 2048-2053.

[10] P. Ioannou and J. Sun, Robust Adaptive Control. : Prentice Hall, 1996.

[11] R. Sonntag and C. Borgnakke, Introduction to Engineering Thermodynamics. New York: Wiley, 2002.
[12] V. Tsourapas, "Modeling, optimization, and control of a combined heat and power fuel processor for hydrogen generation from natural gas," M.S. Thesis, Dept. Mech. Eng., Univ. Michigan, Ann Arbor, 2005.

[13] M. Naghshineh and M. Keshumiri, "Actuator saturation avoidance in overactuated systems," in Proc. IEEE/RSJ Int. Conf. Intell. Robots, 2004, pp. 3613-3618.

[14] K. Ohishi, H. Nozawa, and T. Miyazaki, "Redundant manipulator control with autonomous consideration algorithm of torque saturation," in Proc. ISIE, 1999, pp. 145-150.

[15] O. Harkegard, "Resolving actuator redundancy-Control allocation vs. Linear quadratic control," presented at the ECC, Cambridge, U.K., 2003.

[16] M. Bodson, "Evaluation of optimization methods for control allocation,” J. Guid., Control, Dyn., vol. 25, no. 4, pp. 703-711, Jul.-Aug. 2002.

[17] J. S. Freudenberg, A First Graduate Course in Feedback Control. Ann Arbor: Michigan Art and Architecture Engineering Library, 2005.

[18] Phillip H. Sherrod, Brentwood, TN, "DTREG predictive modeling software," 2004. [Online]. Available: http://www.dtreg.com

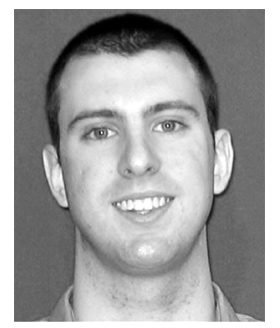

Chris Vermillion received the B.S.E. degree in aerospace and mechanical engineering and the M.S. degree in electrical engineering from the University of Michigan, Ann Arbor, in 2004 and 2005, respectively, where he is currently pursuing the $\mathrm{Ph} . \mathrm{D}$. degree in electrical engineering and computer science, working jointly with Toyota Technical Center (Ann Arbor, MI) on advanced powertrain research and development.

His research interests include advanced powertrain control, modular control strategies, and optimal control, with specific application to model predictive control allocation for overactuated systems.

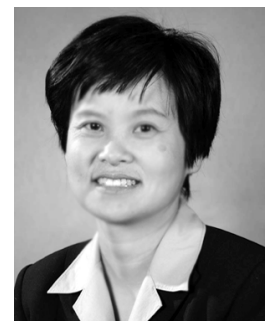

Jing Sun (F'04) received the Ph.D. degree from the University of Southern California, Los Angeles, in 1989, and the B.S. and M.S. degrees from University of Science and Technology of China, Hefei, in 1982 and 1984 , respectively.

From 1989 to 1993, she was an Assistant Professor with the Electrical and Computer Engineering Department, Wayne State University, Detroit, MI. In 1993, she joined Ford Research Laboratory, where she worked in the Powertrain Control Systems Department. After spending almost 10 years in industry, she came back to academia and joined the faculty of the College of Engineering, University of Michigan, Ann Arbor, in 2003, where she is now a Professor with the Department of Naval Architecture and Marine Engineering and the Department of Electrical Engineering and Computer Science. Her research interests include system and control theory and its applications to marine and automotive propulsion systems. She holds over 30 U.S. patents and has coauthored a textbook on robust adaptive control.

Prof. Sun was a corecipient of the 2003 IEEE Control System Technology Award.

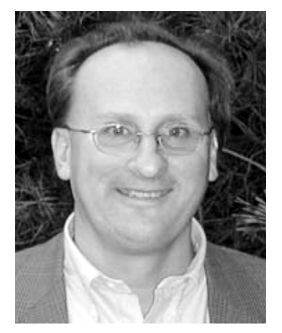

Ken Butts received the B.E. degree in electrical engineering from General Motors Institute (now Kettering University), Flint, MI, the M.S. degree in electrical engineering from the University of Illinois, Urbana-Champaign, and the Ph.D. degree in electrical engineering systems from the University of Michigan, Ann Arbor.

$\mathrm{He}$ is an Executive Engineer with the Powertrain and Chassis Division, Toyota Motor Engineering and Manufacturing North America, Ann Arbor, MI, where he is investigating methods to improve engine control development productivity. 